Regards sur l'économie allemande

Bulletin économique du CIRAC

$110 \mid 2013$

Varia

\title{
Apprentissage : des jeunes pragmatiques et heureux
}

\section{Isabelle Bourgeois}

\section{(2) OpenEdition}

1 Journals

Édition électronique

URL : http://journals.openedition.org/rea/4595

DOI : 10.4000/rea.4595

ISSN : 1965-0787

Éditeur

CIRAC

\section{Édition imprimée}

Date de publication : 15 octobre 2013

Pagination : 37-38

ISSN : 1156-8992

\section{Référence électronique}

Isabelle Bourgeois, «Apprentissage : des jeunes pragmatiques et heureux », Regards sur l'économie allemande [En ligne], 110 | octobre 2013, mis en ligne le 16 octobre 2013, consulté le 15 septembre 2020. URL : http://journals.openedition.org/rea/4595

Ce document a été généré automatiquement le 15 septembre 2020.

(c) CIRAC 


\title{
Apprentissage : des jeunes pragmatiques et heureux
}

\author{
Isabelle Bourgeois
}

1 A l'heure où tant de jeunes Européens sont au chômage et désespèrent de l'avenir, la joie de vivre des jeunes Allemands qui abordent ou achèvent un apprentissage dans le système dual fait exception. Ils savent ce qu'ils veulent, sont conscients des réalités du monde dans lequel ils vivent et, s'ils rêvent de s'épanouir au travail (!), ils ne se bercent pas d'illusions non plus.

\section{Etude McDonald's sur l'apprentissage}

Dans le prolongement des «Etudes sur la jeunesse de Shell » réalisées depuis 60 ans pour le compte de Shell Allemagne par l'institut TNS Infratest Sozialforschung (Munich) et accompagnées scientifiquement par l'Université de Bielefeld, la société McDonald's Allemagne vient de publier une étude commanditée à l'Institut für Demoskopie d'Allensbach (IfD). Si les études Shell analysent l'évolution des aspirations et des modes de vie des 12-25 ans (voir: www.cirac.u-cergy.fr/colloques_etudes_wp/ Shell_Jugendstudie.pdf), l'étude de l'IfD réalisée en mai/juin 2013 porte sur les 15-24 ans. Elle se penche exclusivement sur les préoccupations des collégiens abordant orientation ou formation professionnelle, des jeunes en apprentissage et des jeunes salariés. Menée par sondage auprès de 3068 jeunes, représentatifs de 9,04 millions de personnes, cette première "Etude McDonald's" (d'autres sont prévues) présente une photographie très fine aussi bien des enjeux économiques et sociaux de l'apprentissage que du vécu de ceux qui en bénéficient. Le titre en résume joliment la tonalité : "Pragmatiques et heureux : les apprentis entre divan et carrière ». 


\section{$62 \%$ des jeunes veulent un métier leur permettant de s'épanouir}

3 Le premier enseignement qu'on en retient, c'est qu'en Allemagne, les jeunes abordent leur avenir professionnel avec confiance: ils sont $71 \%$ dans ce cas. Cette part est particulièrement élevée ( $81 \%$ ) chez ceux qui sont convaincus d'avoir choisi la bonne voie ; ceux qui en doutent ne sont que $41 \%$. Interrogés sur ce qui à leurs yeux compte le plus dans la vie, ils placent en tête: avoir de bons amis (69\%), être en bonne santé (64\%) et « avoir un métier qui me plait et où je peux m'épanouir » (62\%), suivis de la famille $(60 \%)$, la sécurité de l'emploi (58\%) ou d'un couple heureux (56\%). Ces jeunes-là sont visiblement bien intégrés dans la société, qu'il s'agisse de leur environnement familial ou professionnel. Les loisirs (36\%) leur importent nettement moins que leur participation dans la société.

\section{Ils sont conscients de la continuité des politiques}

Peut-être trouve-t-on une réponse au fort taux d'indécis chez les primo-électeurs lors des dernières élections au Bundestag dans ce qu'ils pensent de l'effet de ces dernières sur leur sort personnel. Car, à la question qui leur était posée en ce sens, ils sont $64 \%$ à dire que "l'issue des élections n'aura aucune incidence sur [eux] personnellement», et seulement $15 \%$ à affirmer le contraire. Et plus ils sont entrés dans la vie professionnelle, plus ils sont convaincus de la continuité : chez les apprentis et les jeunes actifs occupés, le taux de réponses atteint $69 \%$.

\section{Les jeunes adhèrent au modèle de société allemand}

5 15-24 ans étant l'âge de transition vers la vie professionnelle, les valeurs évoluent. Ainsi, ce qui importe le plus à ceux qui se trouvent encore dans la phase d'orientation, c'est de "trouver un métier qui me remplisse et que j'aie plaisir à exercer»; les taux de réponses des collégiens, étudiants et apprentis débutants se situent entre $66 \%$ et $69 \%$. Mais dès qu'ils sont entrés dans la vie active, c'est la sécurité de l'emploi qui compte le plus (pour $66 \%$ des actifs occupés et $68 \%$ des apprentis). Confiants dans l'avenir, ces jeunes sont aussi très 'libéraux'. Les deux tiers d'entre eux (63\%) pensent en effet que, "dans notre système économique, la performance individuelle est couronnée de succès et que, en règle générale, les efforts sont récompensés"; seuls $13 \%$ affirment que "chez nous, l'effort n'est pas récompensé». Les plus convaincus sont les plus jeunes (les collégiens, $68 \%$ ) et les étudiants (70\%), ce qui peut s'expliquer par leur statut d'apprenant. Ceux qui sont dans la vie active se montrent un peu moins affirmatifs, mais sont $59 \%$ tout de même à croire en la valeur de l'effort. Le taux de réponses positives est particulièrement élevé chez ceux qui estiment que leur propre situation économique est bonne ou très bonne (74 \%); ceux qui l'estiment (très) mauvaise ne sont que $34 \%$ à partager cette approche.

\section{La catégorie sociale reste un frein à l'ascension}

Dans l'ensemble, 47 \% des sondés évaluent leur situation comme (très) bonne ; 37 \% trouvent que «ça va ». Les jeunes Allemands ne désespèrent donc pas de leur sort, et 
même les jeunes issus de l'immigration sont satisfaits ( $42 \%$ de réponses «(très) bonne » et $37 \%$ de « ça va »). L'ascenseur social semble bien fonctionner, puisque $59 \%$ de tous les sondés et, parmi eux, de $51 \%$ des Allemands issus de l'immigration répondent "(très) bonnes" à la question: "Qu'en pensez-vous : comment se présente la possibilité de grimper sur l'échelle sociale en Allemagne, je veux dire quand par exemple un enfant d'ouvrier veut progresser?». Plus importante que l'origine des parents semble l'appartenance sociale: c'est en bas de l'échelle que l'ascenseur social est considéré comme en panne. Dans cette catégorie, 53 \% répondent « moins bonnes/pas bonnes du tout ».

\section{Travail : le plaisir avant le salaire}

7 Qu'attendent-ils de leur travail? «Que j'y aie du plaisir » (71\%), « que mon emploi soit garanti » $(64 \%)$, «que mon métier corresponde à mes capacités et à mes aspirations » $(50 \%)$ - voilà le trio de tête. Viennent ensuite, en $8^{\mathrm{e}}$ position : « un travail qui se laisse bien concilier avec ma vie privée et familiale » $(35 \%)$, et en $9^{\mathrm{e}}$, « un salaire calé sur la performance » $(34 \%)$.

\section{Un jeune sur deux ne regrette pas son choix}

8 Enfin, à la différence de leurs homologues français, les collégiens allemands disent qu'ils se réjouissent en pensant à la vie après l'école : $58 \%$ « beaucoup/pas mal ». $37 \%$ répondent « comme ci, comme ça », et seulement $4 \%$ «pas vraiment/ pas du tout». Les optimistes sont particulièrement nombreux chez ceux qui ont choisi l'apprentissage et qui savent déjà quel métier ils veulent apprendre $(75 \%)$ comme chez ceux qui veulent faire des études (62\%), sachant que les qualifications supérieures offrent les meilleures perspectives d'évolution et de rémunération. Mais l'écart avec ceux qui choisissent l'apprentissage est très faible : ces derniers sont $58 \%$ à se montrer optimistes. Cela dit, comme tous les jeunes confrontés au choix de la meilleure préparation à la vie professionnelle, les Allemands aussi éprouvent des difficultés : $56 \%$ des collégiens interrogés disent qu'ils trouvent ce choix « (très) difficile ». Les garçons (61 \%) plus que les filles ( $42 \%$ ), les futurs étudiants ( $58 \%$ ) plus que les futurs apprentis ( $46 \%$ ). Mais une fois en apprentissage, ils pensent largement avoir fait le bon choix : $44 \%$ disent qu'ils sont occupés exactement dans l'entreprise qu'ils voulaient et $40 \%$ dans le métier ou domaine souhaité. Alors pourquoi changer ? $83 \%$ des apprentis disent ne jamais y avoir pensé, et $54 \%$ aimeraient être recrutés ensuite par leur entreprise formatrice. Une fois dans la vie active, ils ne regrettent pas leur choix : $44 \%$ affirment être employés dans le métier/domaine, $34 \%$ dans l'entreprise qu'ils voulaient. Et ensuite? Les deux tiers des apprentis et des jeunes salariés sont optimistes quant à leurs perspectives d'avenir.

\section{L'étude McDonald's s'insère dans un effort collectif de modernisation du système dual}

9 Cette étude, qui bénéficie de l'accompagnement scientifique de la Hertie School of Governance, est une des nombreuses contributions qu'apportent actuellement toutes les parties concernées (des pouvoirs publics aux syndicats en passant par les 
fédérations professionnelles, les CCI ou les milieux scientifiques) pour moderniser et rendre plus inclusif encore le système dual allemand. Une des dernières initiatives en date est un memorandum publié conjointement en septembre par des réseaux de DRH (Deutsche Gesellschaft für Personalführung, DGFP, et Human Resources Alliance). Il s'intéresse tout particulièrement à l'intégration des jeunes en difficulté qui ne trouvent pas de place d'apprentissage, notamment parce qu'ils ne maitrisent pas les basic skills, ou qui bénéficient de ce fait de mesures de pré-qualification (Zukunft durch Berufsabschluss - Gegen Bildungsarmut). C'est là une réponse apportée au vieillissement démographique - dans une optique à la fois de cohésion sociale, de hausse des qualifications et donc de compétitivité. (IB)

INDEX

Mots-clés : apprentissage, formation professionnelle, jeune, groupe social, marché du travail, emploi, société 